\title{
100 NM THICK ALUMINUM NITRIDE BASED PIEZOELECTRIC NANO SWITCHES EXHIBITING 1 MV THRESHOLD VOLTAGE VIA BODY-BIASING \\ Nipun Sinha ${ }^{l^{*}}$, Zhijun Guo ${ }^{l}$, Valery V. Felmetsger ${ }^{2}$ and Gianluca Piazza ${ }^{l}$ \\ ${ }^{1}$ University of Pennsylvania, Philadelphia, Pennsylvania, USA \\ ${ }^{2}$ Tegal Corporation, San Jose, California, USA
}

\begin{abstract}
This paper reports on the first demonstration of aluminum nitride (AlN) piezoelectric logic switches that were fabricated with ultra-thin (100nm) AlN films and exhibit a $1 \mathrm{mV}$ threshold voltage via the body-biasing scheme. The application of a relatively low $(<6 \mathrm{~V})$ fixed potential to the body terminal of a 4-terminal switch has resulted in a repeatable threshold voltage of $1 \mathrm{mV}$. The nanoswitch has been cycled to $>10^{9}$ cycles and, although the contact resistance was found to be high $(\sim 1 \mathrm{M} \Omega)$, the nano-films have functioned throughout to show high piezoelectric nano-film reliability.
\end{abstract}

\section{INTRODUCTION}

With the continuous scaling of the transistor the CMOS industry has recognized the emergence of some key problems that are proving to be serious obstacles to further miniaturization. Some of the major hurdles that need to be overcome involve the source-to-drain leakage in the standby state, the gate leakage because of ultra-thin dielectric layers, the inability to reduce operating power, the variation of threshold voltages over a wafer and the increasing effect of parasitics on the device performance. With miniaturization, transistors have become faster, but the gain in speed has come at a penalty in terms of standby power consumption. Also, with the CMOS transistors already switching in less than few nanoseconds, power is becoming a more relevant aspect to consider than speed. Keeping all these factors in mind the International Technology Roadmap for Semiconductors
(ITRS) [1] has emphasized the need to develop alternate devices, like NEMS switches, that will consume less power in the standby state and will help in minimizing the transistor operating voltages.

Mechanical switches have been a topic of research and investment for few decades. They have nearly zero standby leakage due to the presence of an air gap between the source and the drain terminals. In addition, they are characterized by a very sharp transition between their standby and on states. This transition is not governed, as in a semiconductor, by the modulation of carriers in the channel, but by the actual mating of contacts due to mechanical motion. Therefore, mechanical switches exhibit a subthreshold slope that is orders of magnitude lower than that of CMOS devices. Because of these characteristics they are the ideal candidate to lower power consumption in the standby state and operating voltages. Most of the mechanical switches developed to date have utilized electrostatic [2-3], magnetic [4], thermal [5] or piezoelectric [6-9] actuation mechanisms. These mechanical switches have not been commercialized on a large scale as they are not as reliable and as fast as the semiconductor transistors. Micromechanical switch reliability has been limited by the very stringent requirements on the on-resistance as dictated by radio frequency (RF) applications (i.e. few Ohm of contact resistance), which have so far been the most attractive for microswitches. When we consider the same devices for implementation of mechanical computing/logic, the main design challenge resides not in the loss due to the contact resistance but the speed of operation. According to these new guidelines a mechanical switch can operate with contact

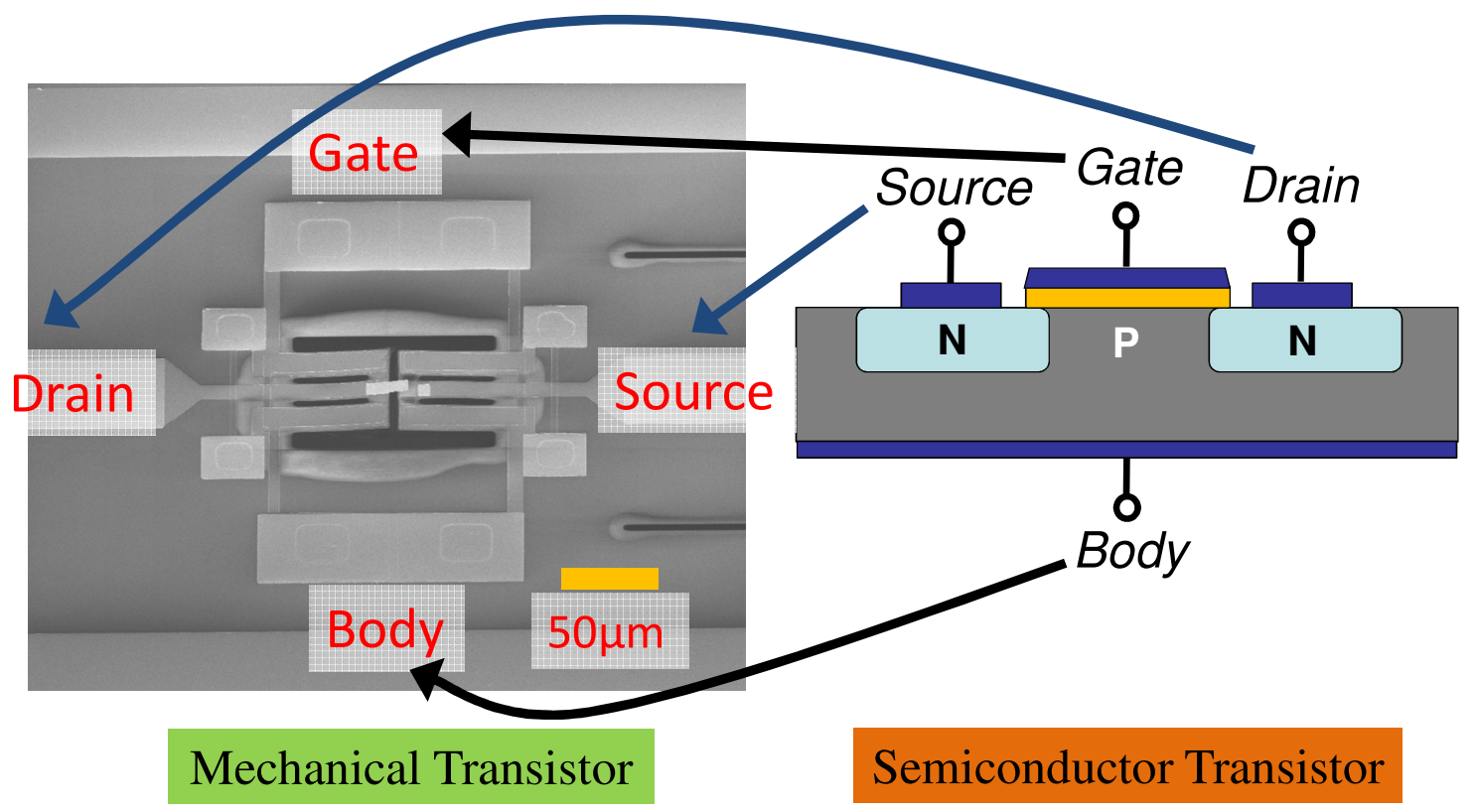

Figure 1: SEM of a nano-film based three-finger dual-beam AlN switch illustrating the source, drain, gate and body bias terminals. This nano-switch is being termed as a Mechanical Transistor. The figure above also shows its similarity with a Semiconductor Transistor shown on the right of the figure. 
resistances in excess of $10 \mathrm{k} \Omega$, therefore significantly relaxing the reliability challenges that exist with hot switching of large currents. Nonetheless, significant issues arise in the miniaturization of the mechanical switches to achieve higher frequencies of operation (approaching 100s of MHz).

For these reasons, there has been a renewed interested in the development of fast electrostatic [10-12] and piezoelectric [9, 1314] nano switches. These implementations have utilized the concept of a 4-terminal device for tuning the threshold voltage of the switch and reducing the dynamic power consumption. Figure 1 shows an SEM of a piezoelectric nano-switch and highlights its similarity with a 4-terminal MOSFET. Though electrostatic actuation has been the most common method of implementing mechanical switches, we have used piezoelectric actuation as it is linear and can easily produce a pull-off force. Amongst the various piezoelectric materials being commonly used for research, Lead Zirconate Titanate (PZT) and AlN have already been employed for fabricating mechanical switches [6-9]. Nonetheless, PZT has the drawback that it is incompatible with present day CMOS foundries. AlN, on the other hand, is post-CMOS compatible, has higher dielectric strength, is amenable to scaling to the nano-dimensions [15] and is easy to process. AlN MEMS switches have already been used to demonstrate logic elements like NOT [13], NAND and NOR [14] gates and prove the concept of body biasing in 4-terminal piezoelectric devices.

The mechanical switches presented in this work have structural AIN layers that have been scaled in thickness to $100 \mathrm{~nm}$, exhibit a low subthreshold slope $(\sim 0.033 \mathrm{mV} / \mathrm{dec})$ and the ability to operate at lower supply voltages than CMOS. The use of low supply voltages translates in greatly reduced power consumption with energy per operation in the order of few tens of aJ. The AlN film is an order of magnitude thinner than the films used for making switches in [13], operate with lower body-biasing voltages $(6$ vs. $20 \mathrm{~V})$, and especially are capable of a threshold voltage of $1 \mathrm{mV}$ (w.r.t. to $30 \mathrm{mV}$ in [13]). The demonstration of these nanoswitches represents a significant step forward towards the implementation of ultra-low active power mechanical computing.

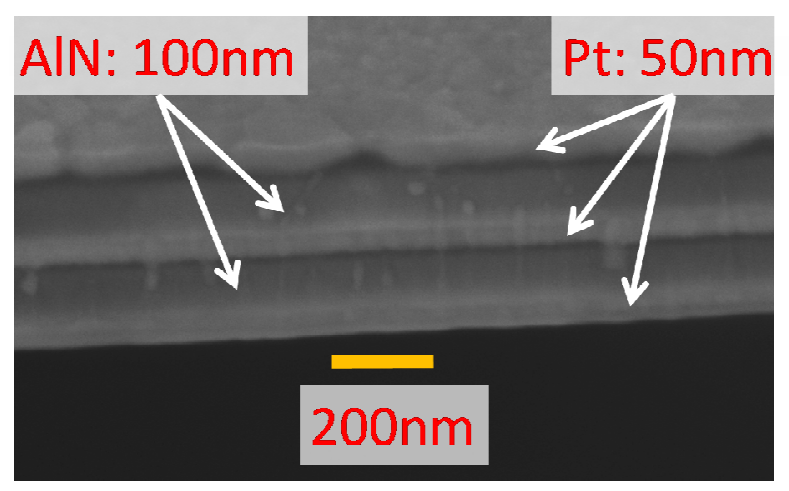

Figure 2: The zoomed-in SEM of the cross-section of the nanoactuator shows the AlN nano-films (100nm thick) and the sandwiching Pt films (50 $\mathrm{nm}$ thick).

\section{NANO-SWITCH AND BODY BIASING}

The piezoelectric switch shown in Fig. 1 is a miniaturized version of the switch implemented earlier in [7, 13-14]. It is a three-finger dual beam switch that has been fabricated using two layers of $100 \mathrm{~nm}$ thick AlN sandwiched by three layers of $50 \mathrm{~nm}$ thick Platinum (Pt) (Fig. 2). The AlN nano-films have been deposited by Tegal Corporation, CA. The authors, in collaboration with Tegal Corp have already demonstrated that the piezoelectric properties of $100 \mathrm{~nm}$ AlN nano-films are comparable to those of thicker AlN films. [15].

The switches presented in this work have been fabricated using an 8-mask process on silicon wafers that is based on the same steps presented in [14].

In this work, the scaling of the AlN films and the consequently improved control over the actuation voltage has enabled us to demonstrate a decrease in the threshold voltage of the switches. This threshold voltage control has been achieved by employing the body-biasing method [13-14]. Figure 3 explains how the body biasing scheme can be used for accurate control of the threshold voltage. In Fig. 3(a), the commonly used grounded actuation mode (body terminal grounded) is shown. In this mode the $\mathrm{V}_{\text {th }}$ and the Ground are able to generate the electric field desired for actuation in the piezoelectric film. This same electric field (as shown in Fig. 3(b)) can be obtained by fixing a bias voltage on the set of electrodes that are grounded in Fig. 3(a). By introducing this body-bias the required electric field for actuating the switch is achieved by applying a gate voltage of just $+100 \mathrm{mV}$ on the set of electrodes that required a gate voltage of $\mathrm{V}_{\text {th }}$ in Fig. 3(a). In this example, the threshold voltage was selected to be $100 \mathrm{mV}$, but can be adjusted to any arbitrary value by controlling the value of the body-bias, as shown in [13-14].

In this work, $\mathrm{V}_{\text {th }}$ was controlled to be $1 \mathrm{mV}$. Achievement of a $1 \mathrm{mV}$ threshold voltage is greatly attributed to the scaling of the film thickness and the subsequent decrease in the required bodybias voltage. Switches fabricated with $100 \mathrm{~nm}$ AlN films need a body bias $\leq 6 \mathrm{~V}$ whereas the devices made with $1 \mu \mathrm{m}$ AlN needed a bias of $\leq 20 \mathrm{~V}$ [13] for low threshold voltage actuation. In this specific demonstration, the change in electric filed caused by $1 \mathrm{mV}$ is 30 times higher for the $100 \mathrm{~nm}$ AlN film based switches than for the switches that use the thicker $1 \mu \mathrm{m} \mathrm{AlN} \mathrm{[13].} \mathrm{The} \mathrm{higher}$ sensitivity of electric field to voltage variations is enabled by the scaling of the film thickness, which simultaneously help in reducing the body bias voltage and increase the degree of control over actuation.

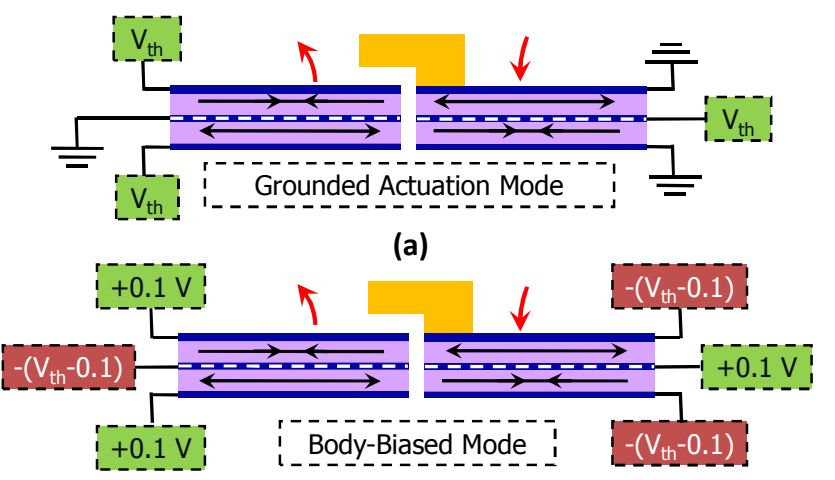

(b)

Gate Voltage

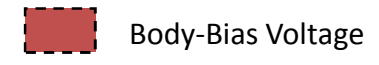

Figure 3: Schematic representation of (a) grounded actuation mode and (b) body-biased actuation mode.

\section{EXPERIMENTAL RESULTS}

The device threshold voltage was measured by monitoring the change in contact resistance while varying the gate voltage. An Agilent E3631A DC power supply was used to apply the body-bias voltage. An Agilent 34401A multi-meter and an Agilent 33250A function generator were both controlled using LabView to step 
through different voltages so as to study the variation of contact resistance with respect to gate voltage and applied body-bias.

Figure 4 shows the variation of the $\mathrm{V}_{\text {th }}$ achieved by changing the body-bias. The plot shows that a threshold voltage of $1 \mathrm{mV}$ has been measured. Switching occurred when the contact resistance suddenly increased to a value that was out of the range of measurement of the multi-meter. The open state value was assumed to be greater than $1 \mathrm{G} \Omega$ according to the specifications of the available multi-meter. From the same experiment, a value of $<0.033 \mathrm{mV} / \mathrm{dec}$ can be estimated for the subthreshold slope. This extracted subthreshold slope is comparable to those measured on similar micromechanical switches. The variation of the threshold voltage displayed in Fig. 4 is the first experimental validation that body-biasing functions, as expected, in AlN nano-films.

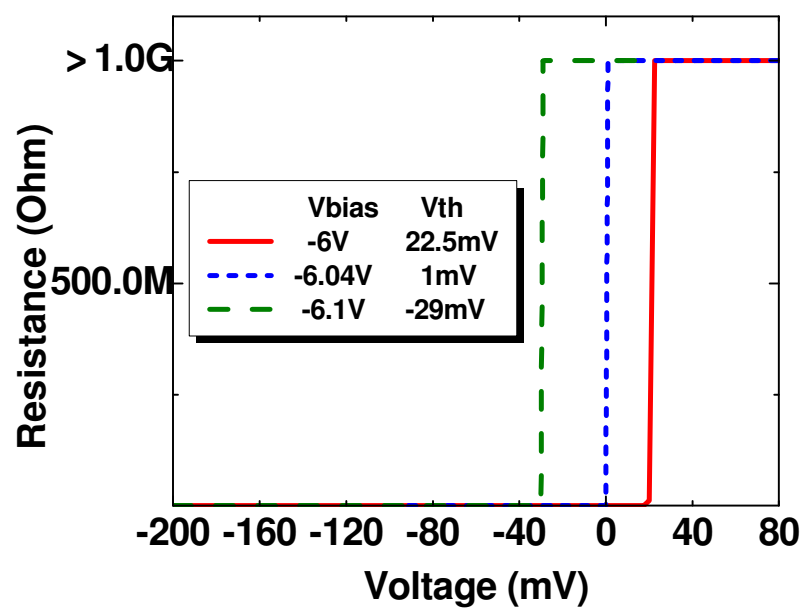

Figure 4: The ability to use body-biasing to shift the threshold voltage is demonstrated. $1 \mathrm{mV}$ threshold was measured by stepping through the applied gate voltage at $0.1 \mathrm{mV}$ steps.

These nano-film based switches can be actuated with voltages much lower than the breakdown value of the film, which was found to be $\sim 12 \mathrm{~V}$. The use of voltages lower than the breakdown is associated with low leakage currents flowing from the gate to the body terminal and assures that low standby power consumption can be attained via the body-biasing technique.

These switches exhibit a contact resistance $(\sim 1 \mathrm{M} \Omega)$ higher than usually expected for metal contact switches [2-8] (Fig. 5). The possible reasons for this high contact resistance are the low force at the contact and the presence of Titanium Oxide $\left(\mathrm{TiO}_{2}\right)$ at the interface. This layer of $\mathrm{TiO}_{2}$ could have formed by oxidation of the Ti adhesion layer used during the Pt deposition. Figure 6 highlights how the contact resistance varies when the gate voltage is stepped from $-2 \mathrm{~V}$ to $+2 \mathrm{~V}$, i.e. when the contact force is varied. From these results it is evident that the resistance is a strong function of the applied force. The contact force is the highest at the beginning of the sweep where the resistance is $\sim 10 \mathrm{k} \Omega$. As the voltage is increased the contact force decreases and there is a sudden increase in contact resistance to $\sim 200 \mathrm{k} \Omega$. The exact phenomenon for this sudden increase in resistance is not known, but it can be likely attributed to the partial breaking of contact bonds between the two mating surfaces. As the voltage is further increased, the resistance rises up to a value of $\sim 1 \mathrm{M} \Omega$. Past $0.52 \mathrm{~V}$ the switch completely breaks contact and an open is recorded.

The low subthreshold slope permits operating the switch with very low supply voltages and effectively take advantage of the

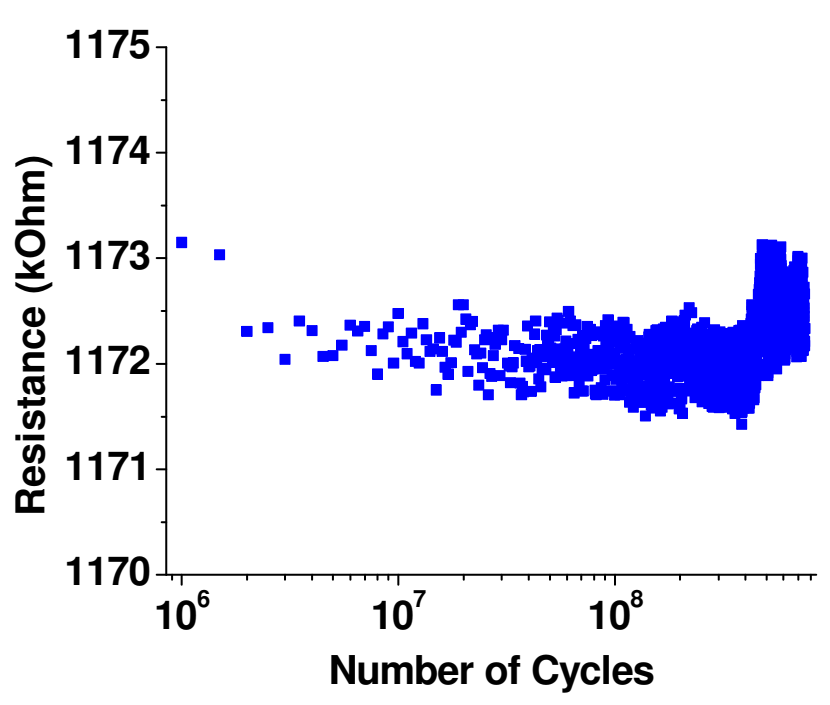

Figure 5: Experimental results for the reliability testing of the AlN nano-switch up to 750 million cycles. The device has been tested up to 1 billion cycles before if failed closed. The device was tested at a speed of $500 \mathrm{kHz}$ with a body bias of $4.6 \mathrm{~V}$ and a square wave of $\pm 2 \mathrm{~V}$.

repeatable $1 \mathrm{mV} \mathrm{V}_{\text {th }}$ demonstrated with these nanoscaled devices. The high value of the recorded contact resistance $(\sim 1 \mathrm{M} \Omega)$, though, constitute a possible limitation on the maximum frequency of operation of these devices because of both noise and electrical time constant considerations. The use of other contact pairs with different adhesion energies that will provide for values of contact resistance of few 10 s of $\mathrm{k} \Omega$ are under investigation.

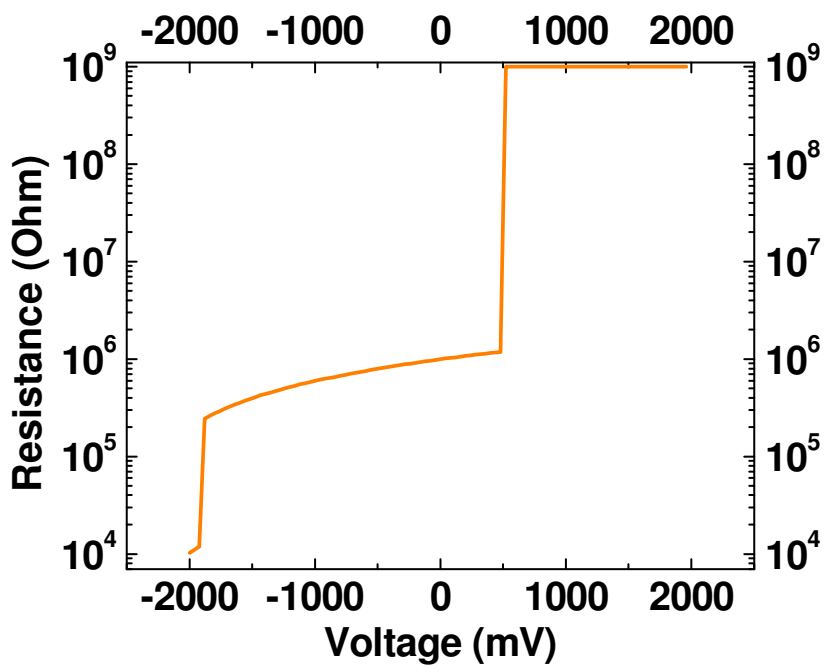

Figure 6: Plot of a single voltage sweep, showing the variation of contact resistance as a function of the gate voltage. The contact resistance is a strong function of the applied force.

The nano-switch was also subjected to reliability testing to study the effect of fast and long term cycling on the Pt-Ti-Pt contact and the nano-piezoelectric film. The resonance frequency of the actuator beam was derived to be $\sim 110 \mathrm{kHz}$ by means of finite element analysis in COMSOL Multiphysics. For the purpose of rapidly testing for a large number of cycles the beams were 
actuated above their resonance frequency, but far from any higher order modes. This was done to minimize the cross-coupling between the intended mode of actuation and other higher order modes of vibration.

Figure 5 shows the results of reliability testing of the switch operated at $500 \mathrm{kHz}$ with a body- bias of $4.6 \mathrm{~V}$ and a square wave form of $\pm 2 \mathrm{~V}$ up to 750 million cycles. Cumulatively, the switch was tested for $>10^{9}$ cycles after which it failed in the closed position. This data shows that the piezoelectric nano-film functioned throughout the testing demonstrating very high nanofilm reliability. Contact reliability greater than $10^{9}$ cycles will have to be demonstrated. Investigations on the contact wearing and its tribological properties are ongoing.

\section{CONCLUSIONS}

In summary, the first AlN piezoelectric switches that employ $100 \mathrm{~nm}$ thick AlN films have been demonstrated. These switches validate the use of the body-biasing technique at the nano-scale. As the thickness of the films has been scaled to the nano-dimensions the body bias voltages have been lowered to $<6 \mathrm{~V}$ and a better control over the actuator motion has been demonstrated. This enhanced control over switching translates into a threshold voltage of $\sim 1 \mathrm{mV}$ and the ability to potentially operate with supply voltages of few $10 \mathrm{~s}$ of $\mathrm{mV}$. Such supply scaling translates in several orders of magnitude reduction in active power consumption. Further scaling of these devices in the lateral dimensions will lead to the development of nanomechanical logic elements that have very low capacitance and reliably operate with extremely low supply voltages.

\section{ACKNOWLEDGEMENTS}

This work was supported by the DARPA NEMS program. The authors would like to acknowledge the help of the staff of the Wolf Nanofabrication Facility at the University of Pennsylvania and the PRISM Micro/Nano Fabrication Laboratory at Princeton University. The authors would also like to thank Mr. T. S. Jones for his help with LabView programming.

\section{REFERENCES}

[1] International Technology Roadmap for Semiconductors 2007 Edition, Austin, TX, Sematech.

[2] P. M. Zavracky, S. Majumder and N. E. McGruer, "Micromechanical switches fabricated using nickel surface micromachining," Jour. of MEMS, vol.6, no.1, pp.3-9, Mar 1997.

[3] C. Bozler, R. Drangmeister, S. Duffy, M. Gouker, J. Knecht, L. Kushner, R. Parr, S. Rabe, and L. Travis, "MEMS microswitch arrays for reconfigurable distributed microwave components", IEEE MTT-S International Microwave Symposium Digest, Boston, MA (June 2000), pp. 153-156.

[4] M. Ruan, J. Shen, and C. B. Wheeler, "Latching Micromagnetic Relays", Journal of MEMS, 10/2001, pp. 511-517.

[5] R. D. Streeter, C. A. Hall, R. Wood, and R. Madadevan, "VHF high-power tunable RF bandpass filter using microelectromechanical (MEM) microrelays", Int. J. RF Microwave CAE, 11/5/2001, pp. 261-275.

[6] R. Mahamameed, N. Sinha, M. B. Pisani, and G. Piazza, "Dual Beam Actuation of Piezoelectric AIN RF MEMS Switches Monolithically Integrated with AlN Contour-mode Resonators," Journal of Micromech. Microeng. 18, 105011, 2008.

[7] N. Sinha, R. Mahameed, C. Zuo, G. Piazza, "Integration of AlN micromechanical contour-mode technology filters with three-finger dual beam AlN MEMS switches," Frequency Control Symposium, 2009 Joint with the 22nd European Frequency and Time forum. IEEE International, pp.1-4, 20-24 April 2009.

[8] H. C. Lee, J. H. Park, J. Y. Park, H. J. Nam and J. U. Bu "Design, fabrication and RF performances of two different types of piezoelectrically actuated Ohmic MEMS switches", Journal of Micromech. Microeng., 15(2004), pp. 2098-2104.

[9] D. C. Judy, J.S. Pulskamp, R. G. Polcawich and L. Currano, "Piezoelectric Nanoswitch" $22^{\text {nd }}$ IEEE International Conference on Micro Electro Mechanical Systems, 2009, pp.591-594, 25-29 Jan. 2009.

[10] R. Nathanael, V. Pott, H. Kam, J. Jeon, and T.-J. King Liu, "4-terminal relay technology for complementary logic," in IEDM Tech. Dig., 2009, pp 223-226.

[11] H. Kam, V. Pott, R. Nathanael, J. Jeon, E. Alon, and T.-J. King Liu, "Design and reliability of a micro-relay technology for zero-standby-power digital logic applications," in IEDM Tech. Dig., 2009, pp. 809-812.

[12] H-H Yang, J. O. Lee and J-B Yoon, "Maneuvering Pull-in Voltage of an Electrostatic Micro-switch by Introducing a Pre-charged Electrode," IEEE IEDM 2007, Washington DC, USA, pp. 439-442.

[13] N. Sinha, T. S. Jones, Z. Guo and G. Piazza, "Body-Biased Complementary Logic Implemented Using AlN Piezoelectric MEMS Switches", 2009 IEEE International Electron Devices Meeting (IEDM 2009), Baltimore, MD, USA, December 2009, pp. 813-816.

[14] N. Sinha, T. S. Jones, Z. Guo and G. Piazza, "Demonstration of Low Voltage and Functionally Complete Logic Operations Using Body-Biased Complimentary and Ultra-Thin AlN Piezoelectric Mechanical Switches," The 23rd IEEE Conference on Micro Electro Mechanical Systems (MEMS 2010), Hong Kong, January 2010, pp. 751-754.

[15] N. Sinha, G. E. Wabiszewski, R. Mahameed, V. V. Felmetsger, S. M. Tanner, R. W. Carpick, and G. Piazza, "Piezoelectric Aluminum Nitride Nanoelectromechanical Actuators,” Appl. Phys. Lett. 95, 053106 (2009).

\section{CONTACT}

*Nipun Sinha, tel: +1-215-573-3276; nipun@ seas.upenn.edu 